\title{
Duodenal-Jejunal Bypass Surgery Stimulates the Expressions of Hepatic Sirtuin 1 and 3 and Hypothalamic Sirtuin 1
}

\author{
Eunyoung $\mathrm{Ha}^{1}$, Jong Yeon Kang' ${ }^{1}$ Kyung Sik Park', Youn Kyoung Seo ${ }^{2}$, Tae Kyung $\mathrm{Ha}^{3, *}$ \\ ${ }^{1}$ Department of Biochemistry, Keimyung University School of Medicine, Daegu; Departments of ${ }^{2}$ Anatomy and Cell Biology and ${ }^{3}$ Surgery, Hanyang University College \\ of Medicine, Seoul, Korea
}

Background: Sirtuins mediate metabolic responses to nutrient availability and slow aging and accompanying decline in health. This study was designed to assess the expressions of sirtuin1 (SIRT1) and sirtuin3 (SIRT3) in the liver and hypothalamus after duodenal-jejunal bypass (DJB) surgery in rats.

Methods: A total of 38 rats were randomly assigned to either sham group $(n=8)$ or DJB group $(n=30)$. DJB group was again divided into three groups according to the elapsed time after surgery (10 weeks, DJB10; 16 week, DJB16; 28 week, DJB28). The mRNA and protein expressions of SIRT1 and SIRT3 in the liver and hypothalamus were measured by reverse transcription polymerase chain reaction, Western blot, and immunohistochemistry analyses. $\mathrm{NAD}^{+} / \mathrm{NADH}$ ratio was also measured.

Results: We found increased mRNA and protein expression levels of SIRT1 in the liver of DJB16 and DJB28 groups compared with those of sham group. The mRNA and protein expressions of SIRT3 in the liver of DJB group increased proportionally to the elapsed time after DJB surgery. The mRNA expression levels of SIRT1 in the hypothalamus increased in DJB16 and DJB28 groups and protein expression levels of SIRT1 in the hypothalamus increased in DJB10, DBJ16, and DJB28 groups compared with sham group. We observed that mRNA and protein levels of SIRT3 in the hypothalamus of DJB group were not changed.

Conclusion: This study proves that DJB increases SIRT1 and SIRT3 expressions in the liver and SIRT1 expression in the hypothalamus. These results suggest the possibility of sirtuins being involved in bypass surgery-induced metabolic changes.

Key words: Duodenojejunal bypass, Life span, Sirtuin1, Sirtuin3

\author{
Received July 23, 2018 \\ Reviewed September 17, 2018 \\ Accepted December 14, 2018 \\ *Corresponding author \\ Tae Kyung $\mathrm{Ha}$ \\ (iD) \\ https://orcid.org/0000-0001-7320-5507 \\ Department of Surgery, Hanyang \\ University College of Medicine, \\ 222-1 Wangsimni-ro, Seongdong-gu, \\ Seoul 04763, Korea \\ Tel: +82-2-2290-8443 \\ Fax: +82-2-2281-0224 \\ E-mail: missurgeon@hanyang.ac.kr
}

\section{INTRODUCTION}

Sirtuins (Sir2-related enzymes) are nicotinamide adenine dinucleotide $(\mathrm{NAD})^{+}$-dependent protein deacetylases that were initially identified as transcription silencer in yeast. ${ }^{1}$ They response to metabolic cues and act as energy sensor relaying the signals to regulate the functions of transcription factors and cofactors via posttranscriptional modification of histone and non-histone proteins. ${ }^{2}$ Of various functions of sirtuins, mediating extension of lifespan in re- sponse to calorie restriction, at least in yeast, is most extensively studied and firmly established. ${ }^{3}$ Overexpression of Sir2 in Saccharomyces cerevisiae prolonged replicative lifespan, which require caloric restriction. ${ }^{4,5}$

Seven mammalian sirtuins with different profiles of actions, substrate affinity, and subcellular compartmentation have been discovered. Of these, sirtuin1 (SIRT1) is most extensively studied followed by sirtuin3 (SIRT3). SIRT1 is widely distributed in mammalian tissues and upregulated when calorie intake is limited. ${ }^{6} \mathrm{Cal}-$

Copyright (C) 2018 Korean Society for the Study of Obesity

(c) This is an Open Access article distributed under the terms of the Creative Commons Attribution Non-Commercial License (http://creativecommons.org/licenses/by-nc/4.0/) which permits unrestricted non-commercial use, distribution, and reproduction in any medium, provided the original work is properly cited. 
orie restriction activates SIRT1, which in turn mediates various cellular metabolic reactions culminating in lifespan extension. Interestingly, a recent study demonstrated that brain specific overexpression of SIRT1 extended the lifespan implicating a role of SIRT1 in the hypothalamus in longevity possibly via modulation of body temperature, appetite, and physical activities. ${ }^{7,8}$ Indeed, hypothalamic SIRT1 regulates feeding and ghrelin response through the agouti-related peptide neurons in ventromedial hypothalamus. ${ }^{9-11}$

SIRT3 is mainly distributed in mitochondria ${ }^{12}$, and also upregulated by calorie restriction. ${ }^{7}$ Lack of SIRT3 in mice has been demonstrated to produce insulin intolerance ${ }^{13}$, and age-related SIRT3 reduction causes insulin resistance and glucose intolerance. ${ }^{14}$ SIRT3 is also known to have effect on cancer cells and neurodegenerative disease including Alzheimer disease, Parkinson disease, and cochlear degeneration. ${ }^{15}$

Bariatric surgery is an effective procedure to cause sustained weight loss and resolution of type 2 diabetes mellitus (T2DM). ${ }^{16}$ Numerous studies to determine the metabolic effects as well as mechanistic details of bariatric surgery have been performed, but to our knowledge, the possible stimulatory effect of bariatric surgery on the expressions of sirtuins has not been examined. Herein, we hypothesized that bariatric surgery would stimulate the expression of sirtuins based on its calorie restriction mimicking effect and explored the potential lifespan extending effect of bariatric surgery by assessing the expressions of SIRT1 and SIRT3 in the liver and the hypothalamus after duodenal-jejunal bypass (DJB) procedure in non-obese rats in a time-dependent manner.

\section{METHODS}

\section{Animal model and diet protocol}

The study group consisted of 38 rats. All rats were kept in individual cages under standard conditions (constant temperature and humidity on a 12-hour light/dark cycle). Eight rats underwent sham operation 12 weeks after birth and 30 rats underwent DJB surgery (DJB group) 12 weeks after birth. Rats of sham group were sampled 10 weeks after operation. Rats in DJB group were sampled 10,16 , and 28 weeks after surgery and regrouped according to the elapsed time after surgery (10 week, DJB10; 16 week, DJB16; 28 week, DJB28). The experimental procedures used in this study were approved by Institutional Animal Care and Use Committee (IACUC No. KM-2016-45) and all procedures performed in studies involving animals were in accordance with the ethical standards of the institution or practice at which the studies were conducted.

\section{Surgical procedures}

All rats were fasted overnight (18 hours) preoperatively. Rats were anesthetized with solution of Zoletil and xylazine (ratio, 2:1; $0.1 \mathrm{~mL} / 100 \mathrm{~g}$; Bayer, Leverkusen, Germany) and placed prone position. A 5-cm abdominal incision was made. Duodenum was ligated $5 \mathrm{~mm}$ distal from pylorus with 5-0 silk. Small bowel $10 \mathrm{~cm}$ distal from Treitz ligament was ligated with 5-0 silk and dissected. A 1-cm incision was made at the starting point of distal jejunum and was anastomosed side to side by 6-0 polydioxanone (PDS) with the body of stomach. Proximal limb was anastomosed side to side by 6-0 PDS $15 \mathrm{~cm}$ distal from gastrojejunal anastomosis. Abdominal incision was closed with 3-0 polyglactin suture (Vicryl; Ethicon, Bridgewater, NJ, USA). Sham operation involved a 5-cm abdominal incision. The incision was closed with 3-0 Vicryl. All rats were allowed access to water right after surgery. Twenty hours after operation, rats were given normal chow diet. Rats were sacrificed 10, 16 , and 28 weeks after operation after overnight fasting. Tissues including liver were harvested for further analyses.

\section{Analysis of mRNA expressions}

The RNA was extracted from hypothalamic and hepatic tissue samples by using TRIzol ( $1 \mathrm{~mL} / 100 \mathrm{mg}$ tissue; Invitrogen, Carlsbad, CA, USA). RNA purity was assessed using a NanoDrop ND1000 UV-Vis spectrophotometer (NanoDrop Technologies, Wilmington, DE, USA). Two micrograms of total RNA were added to each complementary DNA (cDNA) synthesis reaction. RNA was reverse-transcribed into cDNA for 30 minutes at $37^{\circ} \mathrm{C}$ in a reaction mixture containing $200 \mathrm{U}$ of moloney-murine leukemia virus reverse transcriptase (Promega, Madison, WI, USA) and oligo-dT primers, $25 \mathrm{U}$ RNase inhibitor (Promega), $0.5 \mathrm{mM}$ deoxynucleotide triphosphate (Promega), $2 \mu \mathrm{M}$ random hexamer primers. Amplification reaction was performed using prepared cDNA. The following primers were used (Table 1). The mRNA levels of SIRT1 and SIRT3 expressions were determined by normalizing to glyceraldehyde 3-phosphate dehydrogenase expressions. 
Table 1. Oligonucleotides used in quantitative reverse transcription polymerase chain reaction

\begin{tabular}{ll}
\hline Gene & \multicolumn{1}{c}{ Sequence } \\
\hline - Actin & Forward: AGCCATGTACGTAGCCATCC \\
& Reverse: TTCCCTCTCAGCTGTGGTG \\
SIRT1 & Forward: CAGCAACACCTCATGATTGG \\
& Reverse: TCCCACAGGAAACAGAAACC \\
SIRT3 & Forward: GCTGCCAGCAAGGTCTAC \\
& Reverse: CCTTCCACACCCTGGACTA
\end{tabular}

\section{Western blot analysis}

Sampling was performed on anesthetized rats (a peritoneal injection of 2:1 Zoletil and xylazine solution). Tissue samples were frozen in liquid nitrogen and stored at $-70^{\circ} \mathrm{C}$ for subsequent analysis. Hepatic and hypothalamic tissue samples were homogenized in extraction buffer (1X RIPA buffer $2 \mathrm{~mL}+0.2 \mathrm{M}$ PMSF $20 \mu \mathrm{L}+$ PI 20 $\mu \mathrm{L})$, centrifuged at $7,500 \mathrm{rpm}, 4^{\circ} \mathrm{C}$ for 20 minutes. The protein concentrations in the extracts were measured with spectrophotometer. The proteins in the extracts were separated by sodium dodecyl sulfate-polyacrylamide gel electrophoresis gels and transferred to a nitrocellulose membrane. The membranes were blocked with 5\% milk in Tris-buffered saline containing $1 \%$ Triton X-100 (T-BST) buffer at room temperature and then incubated overnight at $4^{\circ} \mathrm{C}$ with anti-SIRT1 (1:1,000; Santa Cruz Biotechnology, Santa Cruz, CA, USA) and anti- $\beta$-actin antibody (1:1,000, Santa Cruz Biotechnology) in blocking solution. The membranes were washed with T-BST buffer and incubated for 1 hour 30 minutes at room temperature with mouse secondary antibodies. The proteins were detected using chemiluminescent substrate reagents (Thermo Scientific, Waltham, MA, USA) and the resulting signals were quantified by using LAS 3000 .

\section{Immunohistochemistry}

Liver tissue was fixed with $10 \%$ formalin, embedded in paraffin, and sectioned. The sections were stained with hematoxylin and eosin for light microscopic morphological examination. For assessment of SIRT1 and SIRT3, 3- $\mu$ m sections were permeabilized in phosphate-buffered saline, incubated in $10 \mathrm{mM}$ sodium citrate buffer with $\mathrm{pH} 6.0$ for $100^{\circ} \mathrm{C}, 10$ minutes, and incubated with antiSIRT1 (1:50 dilution, Santa Cruz Biotechnology) antibody and anti-SIRT3 (1:50 dilution, Santa Cruz Biotechnology) antibody. Sections were then incubated with secondary antibody (1:200 di- lution, Santa Cruz Biotechnology) and followed by staining with diaminobenzidine chromogen (Vector Labs, Burlingame, CA, USA) and counterstaining with hematoxylin. The stained sections were examined under microscopy ( $\times 400$; Nikon, Tokyo, Japan) and all histological assessments were made by a pathologist.

\section{$\mathrm{NAD}^{+} / \mathrm{NADH}$ quantification assay}

Assay kit was purchased from BioVision (Milpitas, CA, USA). $\mathrm{NAD}^{+} / \mathrm{NADH}$ ratio in the liver was determined according to the recommended manufacturer's protocol.

\section{Statistical analysis}

All data are given in terms of relative values and presented as mean and standard deviation. Data were analyzed by Student $t$-test for unpaired comparisons. The accepted level of significance was preset as $P$-value $<0.05$.

\section{RESULTS}

\section{DJB increases hepatic SIRT1 and SIRT3 expressions}

Both mRNA and protein levels of hepatic SIRT1 and SIRT3 increased gradually in a time dependent manner after DJB surgery (DJB16 and DJB28 groups) compared with those in sham group (Fig. 1A, B). Significantly higher expressions of SIRT1 mRNA were observed in DJB16 and DJB 28 groups and those of SIRT3 mRNA in DJB10, DJB16, and DJB28 groups (Fig. 1A). Western immunoblot analysis confirmed that expression levels of SIRT1 and SIRT3 protein were increased in surgery groups (Fig. 1B). Lastly, immunoblot assay also showed increased expressions of both SIRT1 and SIRT3 in DJB28 group compared with those in sham control group (Fig. 1C).

\section{DJB increases hypothalamic SIRT 1 expressions}

We found different expression patterns of hypothalamic SIRT1 and SIRT3 levels in DJB groups. The expression of SIRT1 in the hypothalamus was similar in DJB surgery groups to those in the liver (Fig. 2). DJB surgery increased hypothalamic SIRT1 mRNA and protein expressions. However, SIRT3 mRNA and protein expressions were not increased in DJB surgery groups. 

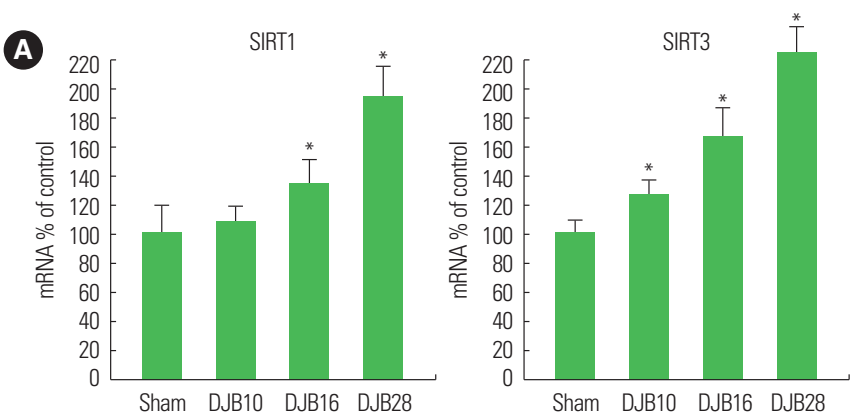

B

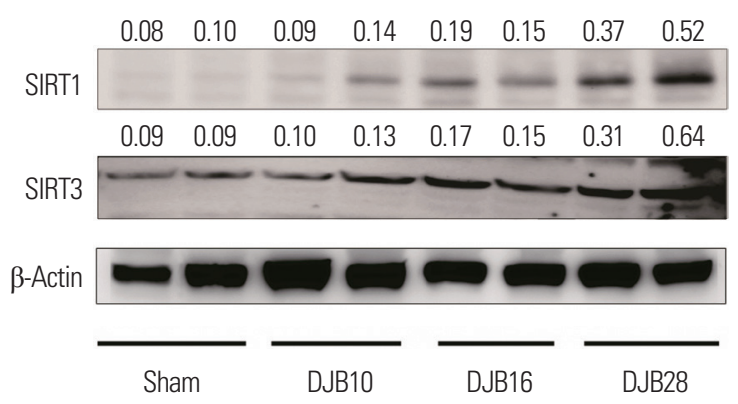

C

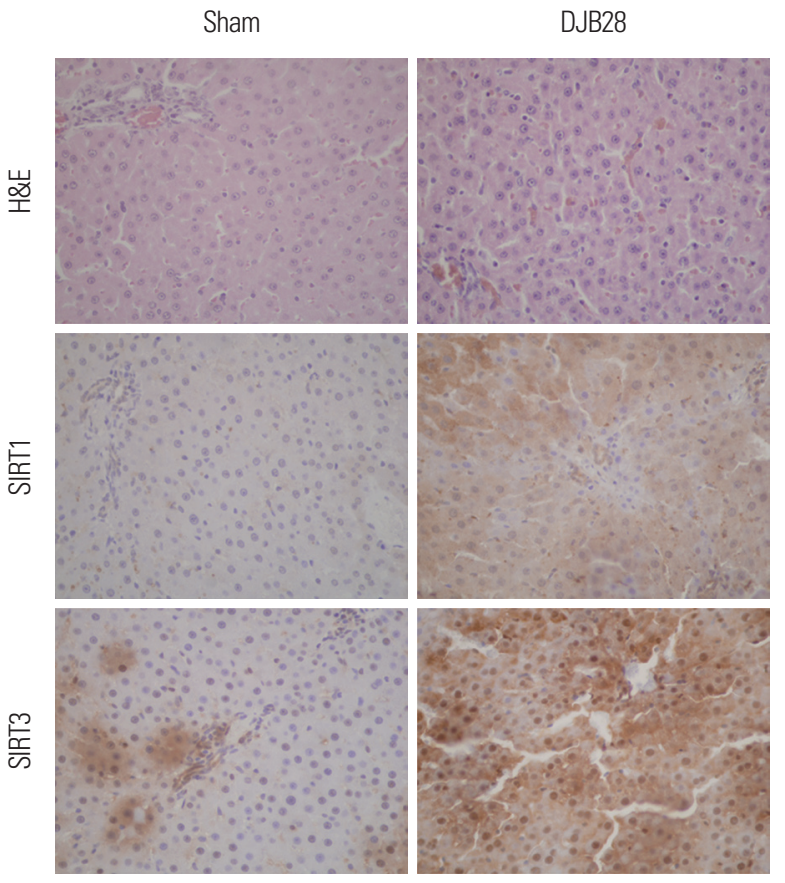

Figure 1. Effects of duodenal-jejunal bypass (DJB) on the expressions of hepatic sirtuin1 (SIRT1) and sirtuin3 (SIRT3) expressions. (A) mRNA levels of SIRT1 and SIRT3 were determined using reverse transcription polymerase chain reaction analysis. (B) Protein levels of SIRT1 and SIRT3 were evaluated by Western immunoblot assay (B) and immunohistochemistry analysis (C). ${ }^{*} P<0.05$ compared with sham control.

\section{DJB increases hepatic $\mathrm{NAD}^{+} / \mathrm{NADH}$ ratio}

SIRT1 protein expression is regulated by the $\mathrm{NAD}^{+} / \mathrm{NADH}$ ratio. Therefore, we tested whether DJB surgery increased $\mathrm{NAD}^{+} / \mathrm{NADH}$ ratio thereby stimulation SIRT1 expressions. And indeed, we found
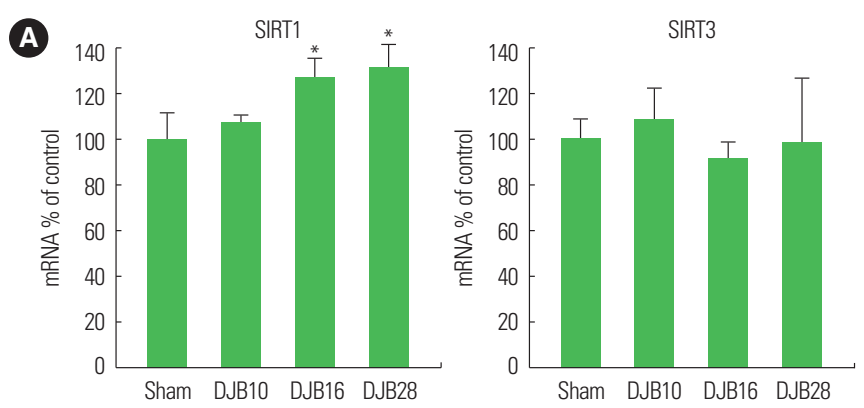

B

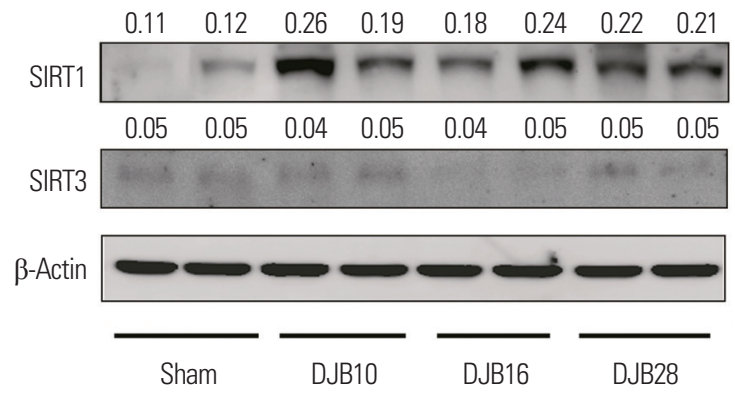

Figure 2. Effects of duodenal-jejunal bypass (DJB) on the expressions of hypothalamic sirtuin1 (SIRT1) and sirtuin3 (SIRT3) expressions. (A) mRNA levels of SIRT1 and SIRT3 were determined using reverse transcription polymerase chain reaction analysis analysis. (B) Protein levels of SIRT1 and SIRT3 were evaluated by Western immunoblot assay. ${ }^{*} P<0.05$ compared with sham control.

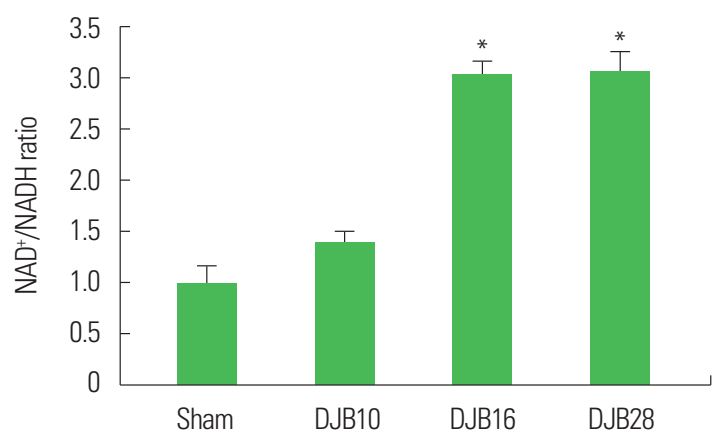

Figure 3. Effects of duodenal-jejunal bypass (DJB) on the hepatic NAD+/NADH ratio. Hepatic $\mathrm{NAD}^{+} / \mathrm{NADH}$ ratio in the liver was determined and compared with sham control. ${ }^{*} P<0.05$ compared with sham control.

DJB surgery groups (DJB16 and DJB28) increased hepatic $\mathrm{NAD}^{+} /$ NADH ratio compared with control sham group (Fig. 3).

\section{DISCUSSION}

Since its first discovery, sirtuins has been the focus of extensive studies. Studies reveal that sirtuins are involved in the regulation of various metabolic processes in the body. Furthermore, sirtuins exert many enzymatic activities from deacetylase, desuccinylase, and 
demaloynylase to adenosine diphosphate-ribosyltransferase, all of which require $\mathrm{NAD}^{+} . \mathrm{NAD}^{+}$is an essential currency of cellular metabolism to which sirtuins have evolved to respond. Cellular $\mathrm{NAD}^{+}$ level affects the activity of sirtuins that suggests the functional connection between sirtuins and $\mathrm{NAD}^{+} .{ }^{17}$

A recent study showed that hepatic SIRT1 expression in old mice is decreased compared with that in young mice and reduced SIRT1 expression is responsible for the impaired body homeostasis..$^{18} \mathrm{An}$ other study also showed association between low SIRT1 expression with obesity and T2DM. ${ }^{19}$ Herein we showed that DJB increased hepatic $\mathrm{NAD}^{+} / \mathrm{NADH}$ ratio, which confirms the connection between hepatic SIRT1 and SIRT3 and NAD ${ }^{+}$levels.

Bariatric surgery has been proven to be an effective treatment that results in sustained weight loss as well as resolution of chronic metabolic diseases such as T2DM. ${ }^{16}$ Given the diverse and important functions of sirtuins in various metabolic processes in the body, we hypothesized that bariatric surgery might have a role in the expression of sirtuins. And we showed in this study that indeed DJB increased hepatic and hypothalamic sirtuins expressions as well as hepatic $\mathrm{NAD}^{+} / \mathrm{NADH}$ ratio. To our knowledge, this study is the first to demonstrate DJB increases sirtuins expressions in the liver and hypothalamus.

Although we proved the stimulatory effect of DJB on the sirtuins expressions, this study is limited in that the underlying mechanism of DJB and functional consequences of increased hypothalamic SIRT1 expression could not be determined. In summary, the current study shows that DJB increases hepatic SIRT1 and SIRT3 and hypothalamic SIRT1. Together with other studies, which show that SIRT1 and SIRT3 have protective metabolic functions against aging and metabolic disorders, DJB-induced expressions of SIRT1 and SIRT3 suggest possible therapeutic effect of bariatric surgery on extending lifespan.

\section{CONFLICTS OF INTEREST}

The authors declare no conflict of interest.

\section{ACKNOWLEDGMENTS}

This work was supported by the research fund of Hanyang Uni- versity (No. HY-201500000003026), Seoul, Korea.

This study was awarded the best poster in 2018 IFSO (The International Federation for the Surgery of Obesity and Metabolic Disorders).

\section{REFERENCES}

1. Guarente L. Sir2 links chromatin silencing, metabolism, and aging. Genes Dev 2000;14:1021-6.

2. Haigis MC, Guarente LP. Mammalian sirtuins: emerging roles in physiology, aging, and calorie restriction. Genes Dev 2006; 20:2913-21.

3. Imai S, Johnson FB, Marciniak RA, McVey M, Park PU, Guarente L. Sir2: an NAD-dependent histone deacetylase that connects chromatin silencing, metabolism, and aging. Cold Spring Harb Symp Quant Biol 2000;65:297-302.

4. Kaeberlein M, McVey M, Guarente L. The SIR2/3/4 complex and SIR2 alone promote longevity in Saccharomyces cerevisiae by two different mechanisms. Genes Dev 1999;13:2570-80.

5. Lin SJ, Defossez PA, Guarente L. Requirement of NAD and SIR2 for life-span extension by calorie restriction in Saccharomyces cerevisiae. Science 2000;289:2126-8.

6. Cohen HY, Miller C, Bitterman KJ, Wall NR, Hekking B, Kessler B, et al. Calorie restriction promotes mammalian cell survival by inducing the SIRT1 deacetylase. Science 2004;305: 390-2.

7. Dietrich MO, Antunes C, Geliang G, Liu ZW, Borok E, Nie Y, et al. Agrp neurons mediate Sirtl's action on the melanocortin system and energy balance: roles for Sirt1 in neuronal firing and synaptic plasticity. J Neurosci 2010;30:11815-25.

8. Satoh A, Brace CS, Ben-Josef G, West T, Wozniak DF, Holtzman $\mathrm{DM}$, et al. SIRT1 promotes the central adaptive response to diet restriction through activation of the dorsomedial and lateral nuclei of the hypothalamus. J Neurosci 2010;30:10220-32.

9. Ramadori G, Fujikawa T, Anderson J, Berglund ED, Frazao R, Michán S, et al. SIRT1 deacetylase in SF1 neurons protects against metabolic imbalance. Cell Metab 2011;14:301-12.

10. Velásquez DA, Martínez G, Romero A, Vázquez MJ, Boit KD, Dopeso-Reyes IG, et al. The central Sirtuin 1/p53 pathway is essential for the orexigenic action of ghrelin. Diabetes 2011; 
60:1177-85.

11. Sasaki T, Kim HJ, Kobayashi M, Kitamura YI, Yokota-Hashimoto $\mathrm{H}$, Shiuchi T, et al. Induction of hypothalamic Sirt1 leads to cessation of feeding via agouti-related peptide. Endocrinology 2010;151:2556-66.

12. Onyango P, Celic I, McCaffery JM, Boeke JD, Feinberg AP. SIRT3, a human SIR2 homologue, is an NAD-dependent deacetylase localized to mitochondria. Proc Natl Acad Sci U S A 2002;99:13653-8.

13. Green MF, Hirschey MD. SIRT3 weighs heavily in the metabolic balance: a new role for SIRT3 in metabolic syndrome. J Gerontol A Biol Sci Med Sci 2013;68:105-7.

14. Hirschey MD, Shimazu T, Jing E, Grueter CA, Collins AM, Aouizerat B, et al. SIRT3 deficiency and mitochondrial protein hyperacetylation accelerate the development of the metabolic syndrome. Mol Cell 2011;44:177-90.

15. Ansari A, Rahman MS, Saha SK, Saikot FK, Deep A, Kim KH. Function of the SIRT3 mitochondrial deacetylase in cel- lular physiology, cancer, and neurodegenerative disease. Aging Cell 2017;16:4-16.

16. Adams TD, Davidson LE, Litwin SE, Kim J, Kolotkin RL, Nanjee MN, et al. Weight and metabolic outcomes 12 years after gastric bypass. N Engl J Med 2017;377:1143-55.

17. Feldman JL, Dittenhafer-Reed KE, Kudo N, Thelen JN, Ito A, Yoshida $\mathrm{M}$, et al. Kinetic and structural basis for acyl-group selectivity and $\mathrm{NAD}(+)$ dependence in sirtuin-catalyzed deacylation. Biochemistry 2015;54:3037-50.

18. Jin J, Iakova P, Jiang Y, Medrano EE, Timchenko NA. The reduction of SIRT1 in livers of old mice leads to impaired body homeostasis and to inhibition of liver proliferation. Hepatology 2011;54:989-98.

19. Song YS, Lee SK, Jang YJ, Park HS, Kim JH, Lee YJ, et al. Association between low SIRT1 expression in visceral and subcutaneous adipose tissues and metabolic abnormalities in women with obesity and type 2 diabetes. Diabetes Res Clin Pract 2013;101:341-8. 